Epidemiology

\title{
Light-intensity physical activity patterns and associated factors in adolescents
}

\author{
Leonardo Alex Volpato $^{1}$ (D), Daniel Zanardini Fernandes ${ }^{1}$ (D), Renan Camargo Correa ${ }^{1}$ (D), \\ Vinícius Muller Reis Weber ${ }^{1}$ (D), Catiana Leila Possamai Romanzini ${ }^{2}$ (D), \\ Enio Ricardo Vaz Ronque ${ }^{1,2}$ (D), Marcelo Romanzini ${ }^{1,2}$ \\ ${ }^{1}$ Programa de Pós-Graduação Associado em Educação Física, Universidade Estadual de \\ Maringá/Universidade Estadual de Londrina, Universidade Estadual de Londrina, PR, Brasil; \\ ${ }^{2}$ Universidade Estadual de Londrina, Departamento de Educação Física, Londrina, PR, Brasil;
}

\author{
Associate Editor: Danilo Rodrigues Pereira da Silva. Universidade Federal de Sergipe, São \\ Cristóvão, SE, Brasil.
}

\begin{abstract}
Aims: This study aimed to measure light-intensity physical activity (LPA) patterns in adolescents according to weekdays and school period and investigated associations between LPA patterns and associated factors. Methods: This is a cross-sectional study with 388 students of both genders, selected from public schools from Londrina city, Parana state. LPA was measured using accelerometry for seven consecutive days and was analyzed by the time spent in very short ( 15 to $30 \mathrm{~s}$ ), short (30 to $60 \mathrm{~s}$ ), intermediate ( 1 to $3 \mathrm{~min}$ ), and long bouts ( $\geq 3 \mathrm{~min}$ ). Results: Approximately $22 \%$ of the total daily time was spent in LPA. The time in LPA accumulated in short or intermediate bouts was higher than the time accumulated in very short or long bouts $(P<0.05)$. Gender was associated with short ( $\beta=2.180 ; 95 \%$ confidence interval [CI], 0.357 to 4.003$)$ and intermediate bouts $(\beta=2.822 ; 95 \% \mathrm{CI}, 0.681$ to 4.964$)$, girls presented higher daily time. Age was inversely associated with very short ( $\beta=-0.925 ; 95 \% \mathrm{CI},-1.676$ to -0.175$)$ and intermediate bouts $(\beta=-1.985 ; 95 \% \mathrm{CI},-3.579$ to -0.390$)$. The total time in LPA and time accumulated in very short, short, and intermediate bouts was higher in the school period when compared to the non-school period $(P<0.05)$.

Conclusion: It could be concluded that the LPA patterns are predominantly composed of time spent in bouts between 30 seconds and three minutes. Gender, age, and school period appear to influence the LPA pattern in adolescents.
\end{abstract}

Keywords: motor activity, epidemiology, accelerometry, students.

\section{Introduction}

It is well documented that physical activity is an important health determinant in adolescence ${ }^{1}$. Physical activity can be classified (based on using the resting metabolic rate as a baseline MET value) by light-intensity physical activity (activities performed between $1.51-2.99$ METs, including activities such as active video games, quiet school work, and slow walking) or moderate-to-vigorous physical activity (activities above 3 METs, including running and playing sports) ${ }^{2}$. Despite being characterized according to these intensities, the recommendation for the daily practice of moderate to vigorous physical activity (MVPA) for at least 60 minutes to achieve health benefits is evident ${ }^{3}$. However, it is estimated that $80 \%$ of adolescents do not meet this recommendation ${ }^{4}$. In addition, adolescents spend a high volume of time in sedentary activities (approximately 10 hours daily $)^{5}$, which is a worrying fact since sedentary behavior is associated with adverse health indicators in this population group ${ }^{6}$.
Recent investigations have focused on light physical activity (LPA) ${ }^{7,8}$ due to its potential to reduce time in sedentary behavior and increase total levels of physical activity ${ }^{9}$. LPA corresponds to a significant portion of the daily behavior ${ }^{10}$ and is characterized between borderline sedentary behavior and MVPA values ${ }^{11}$. Although current global guidelines for the practice of physical activity do not mention LPA, recent evidence suggests health benefits resulting from the practice of LPA in adults, such as reduced mortality and cardiovascular disease indicators ${ }^{7,8}$.

Specifically, in adolescents, the LPA appears to be associated with a series of health benefits, such as the reduction of obesity indicators ${ }^{12,13}$ as well as improvement of some cardiometabolic biomarkers ${ }^{14}$. In addition, the reallocation of sedentary time to LPA seems to be associated with positive results on metabolic and physiological indicators ${ }^{15}$, more favorable indicators of adiposity, cardiorespiratory fitness, and mental health in children and adolescents ${ }^{16}$. LPA patterns can also be associated with health indicators in a particular way. In this 
sense, Blaes et al., ${ }^{17}$ concluded that the number of short LPA bouts $(\leq 3 \mathrm{~min})$ was correlated with lower body fat percentage in boys, while Stone et al, ${ }^{18}$ reported that the frequency of short ( $\geq 4 \mathrm{~s}$ ) and long bouts ( $\geq 5 \mathrm{~min}$ ) from light to very vigorous intensity were associated with a change in endothelial function from rest to post-exercise in boys. Therefore, the boost of LPA through the reduction of sedentary behavior can be characterized as an alternative health strategy in the pediatric population, especially among adolescents who are insufficiently active and/or who accumulate long sedentary behavior time.

Thus, understanding how the LPA pattern manifests itself in adolescents (in terms of volume and bouts) according to gender, periods (weekdays and school hours), and economic context, it is possible to support a more targeted approach to implement interventions with LPA, providing a viable alternative to achieve health benefits. While previous studies have identified that the time spent in sedentary behavior and/or MVPA can vary according to gender $^{19}$, economic level ${ }^{20}$, environment (i.e., school and non-school period), and weekdays (i.e., weekdays and weekends) $^{21}$, LPA characterization in adolescents is still incipient. Thus, the present study aimed: (1) to describe the LPA pattern; (2) to investigate associations between LPA patterns and gender, age, and economic level; (3) to characterize the LPA pattern according to the period of the day (school and non-school period), and weekdays and weekends.

\section{Methods}

\section{Participants}

This was a cross-sectional school-based study carried out with students of both genders selected from public schools in the city of Londrina, Parana state. The sample was composed of adolescents of both genders enrolled in the sixth grade of elementary school. Details about the sample selection process have been previously described ${ }^{5}$. Briefly, ten schools were selected in a stratified way in relation to the geographic region of the municipality (northern, southern, eastern, western regions, and downtown). Conglomerates (classes) were randomly selected until the percentage representation of each school in its respective geographic region was reached. Adolescents were included in the study if they returned the consent form signed by parents or guardians and those enrolled in the sixth grade of elementary school. Participants older than 14 years old, that dropped out during or after the data collection period, those who reported the use of prescribed medicine or were being treated for diseases, if they answered the self-administered questionnaire incorrectly, who refused to use the accelerometer or did not provide valid accelerometer data were excluded from the study sample. The study protocol was approved by the local eth- ics committee (Ethics Committee of the State University of Londrina according to no. 1.281.324.) according to Resolution 466/2012 of the National Health Council on research involving human beings.

\section{Procedures}

Demographic variables (age and gender) were obtained through a self-administered questionnaire. Socioeconomic status was assessed through the Brazilian Association of Research Companies (ABEP) questionnaire ${ }^{22}$, which used a scoring system that assesses the Brazilian families' purchasing power. For analysis purposes, the individual score generated by the instrument was considered, in which the highest score indicates the highest economic level.

The LPA pattern was objectively measured using ActiGraph (ActiGraph, Pensacola, FL, USA) accelerometers, GT3X and GT3X+ models, for seven consecutive days. Each participant received an accelerometer unit and detailed instructions regarding positioning (right side of the hip and level of the anterior iliac crest) and daily use (use during the entire awake period, except during shower and aquatic activities). After the monitoring period, the equipment was collected and recorded data were stored in ActLife software (v. 6.13.3.). Then, the following data reduction criteria were used: a) data were reintegrated from 1-second epoch length to 15 -second epoch length; $b$ ) criterion of 60 minutes of consecutive zeros was used to determine the non-wear time, c) at least eight hours of accelerometer use, including at least four valid days (with at least one weekend day $)^{23}$. After data reduction, estimates of time spent in sedentary behavior, LPA, and MVPA were determined, using cutoff points developed for ActiGraph vector magnitude counts proposed by Romanzini et al. ${ }^{24}$.

Time accumulated in sedentary behavior, LPA, and MVPA was expressed as percentage values ( $\%$ of the total time using an accelerometer). Bouts were defined as uninterrupted LPA periods (drop time $=0$ ). Estimates of accumulated time in bouts were divided into the following time intervals: a) very short ( 15 to $30 \mathrm{~s}$ ); b) short ( 30 to $60 \mathrm{~s}$ ); c) intermediate ( 1 to $3 \mathrm{~min}$ ); d) long ( $\geq 3 \mathrm{~min}$ ). Time spent in bouts was expressed as percentage values in relation to time spent in LPA and calculated to represent the weekly LPA pattern on weekdays (Monday to Friday) and weekends (Saturday and Sunday). The LPA pattern was also considered during the school period (1:15 pm to $5: 50 \mathrm{pm})$ and the non-school period (rest of the day).

\section{Statistical analyses}

To describe the sample, descriptive statistics were used with mean values, standard deviation, and relative frequency. After verifying data normality using the Kolmogorov-Smirnov test, a Student $t$-test for independent samples was performed to detected differences between 
genders. Analysis of variance (ANOVA) was used to compare the different LPA bouts. The Student $t$-test for paired samples was used to analyze differences between total time in LPA, LPA bouts and to compare light-intensity physical activity patterns between weekdays and school periods. Multiple linear regression analysis was conducted to examine associations between variables related to LPA pattern (total time and bouts) and sociodemographic variables (gender, age, and socioeconomic status). Analyses were performed using the IBM SPSS statistical package (version 25.0), with 5\% significance.

\section{Results}

Initially, 980 adolescents were selected to participate in the study, 690 were monitored by accelerometers and 388 obtained valid data (56\%) and were included in the analysis. Sample characteristics are shown in Table 1. The sample consisted mostly of girls $(52.5 \%)$, approximately $70 \%$ of the daily time was spent in sedentary behavior, and boys remain longer time in MVPA when compared to girls $(P<0.05)$.

The LPA pattern is presented in Figure 1. Approximately $22 \%$ (190 $\mathrm{min} /$ day) of the time recorded by accelerometers was spent in LPA. The time spent in LPA in short (30-60 s) or intermediate bouts (1-3 min) was statistically higher than time spent in very short (15-30 s) or long bouts ( $\geq 3 \mathrm{~min})(P<0.05)$. Together, short and intermediate bouts represented almost $70 \%$ of total PA time, while less than $5 \%$ of the time spent in LPA was accumulated in longer bouts ( $\geq 3 \mathrm{~min})$.

The association between LPA patterns and sociodemographic variables (gender, age, and socioeconomic status) is presented in Table 2. Gender was associated with time spent in short ( $\beta=2.180 ; 95 \% \mathrm{CI}, 0.357$ to 4.003 ) and intermediate bouts $(\beta=2.822 ; 95 \% \mathrm{CI}, 0.681$ to 4.964), in which girls presented higher daily time spent in these bouts compared to boys. Age was inversely associated with very short $(\beta=-0.925 ; 95 \% \mathrm{CI},-1.676$ to $-0.175)$ and intermediate bouts $(\beta=-1.985$; 95\% CI, -3.579 to -0.390$)$. No association was observed between the LPA pattern and socioeconomic status.

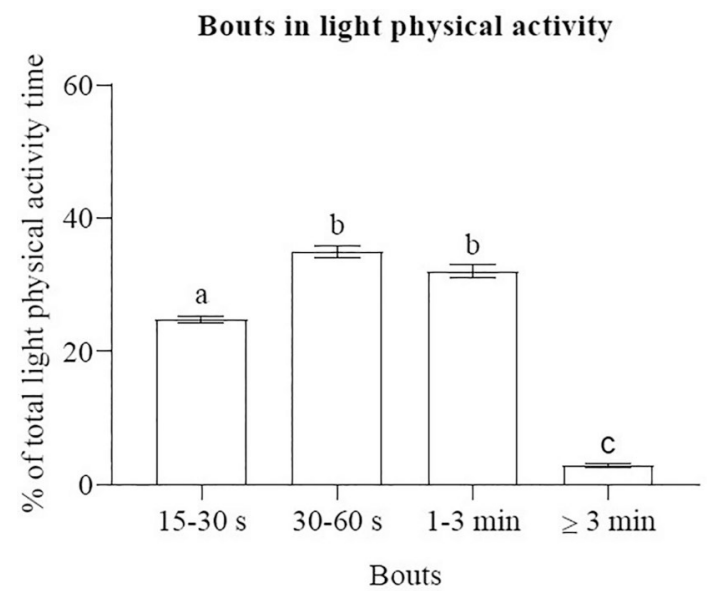

Figure 1 - Pattern of light physical activity in adolescents according to bouts. Note: different letters $=$ significant differences between bouts $(P<0.05)$.

In relation to accelerometer wear time, the total time in LPA did not differ between weekdays and weekends (21.7 vs $22.1 \%, P>0.05$ ), and no differences were observed in LPA bouts in these segments. On the other hand, the total time in LPA $(24.1 \%$ in school and $19.2 \%$ outside school) and LPA time accumulated in very short, short and intermediate bouts was statistically higher during the school period when compared to the non-school period (Figure 2).

\section{Discussion}

The present study found that adolescents spend about $20 \%$ of their waking time on light-intensity activities, which are mostly accumulated from periods ranging from 30 seconds to three minutes. Additionally, it was observed that the LPA pattern was associated with gender, in which girls presented more time spent in short (30 to $60 \mathrm{~s}$ ) and intermediated bouts ( 1 to $3 \mathrm{~min}$ ), and very short (15 to $30 \mathrm{~s}$ ) and intermediate bouts (1 to $3 \mathrm{~min}$ ) were associated with age. Besides, the higher daily time and the LPA pattern were more predominant in the school period when compared to the non-school period. No association was observed with socioeconomic status and the LPA pattern was similar between week and weekend days.

Table 1 - Sample characteristics of the studied population according to gender.

\begin{tabular}{lccc}
\hline Variables & General $(\mathbf{n}=\mathbf{3 8 8})$ & Girls $(\mathbf{n}=\mathbf{2 0 4})$ & Boys $(\mathbf{n}=\mathbf{1 8 4})$ \\
\hline Age (years) & $11.8(0.7)$ & $11.7(0.6)$ & $11.8(0.7)$ \\
Body mass (kg) & $46.6(12.5)$ & $47.4(12.7)$ & $45.8(12.2)$ \\
Height (cm) & $151.7(8.1)$ & $152.4(7.5)$ & $150.6(9.3) *$ \\
Accelerometer wear time (min/day) & $866.7(184.1)$ & $855.5(181.9)$ & $878.9(186.3)$ \\
Sedentary Behavior (\%) & 69.4 & 68.6 & 70.2 \\
MVPA (\%) & 8.7 & 8.6 & $8.9 *$ \\
\hline
\end{tabular}

Abbreviations: MVPA, moderate to vigorous physical activity. ${ }^{*} P<0.05$. 
Table 2 - Association between light physical activity patterns and gender, age, and socioeconomic status from adolescents.

\begin{tabular}{lccc}
\hline LPA pattern & Gender $^{\mathbf{a}} \boldsymbol{\beta}(\mathbf{9 5} \% \mathbf{C I})$ & Age $\left.^{\mathbf{b}} \boldsymbol{\beta} \mathbf{( 9 5 \%} \mathbf{C I}\right)$ & Socioeconomic status $^{\mathbf{c}} \boldsymbol{\beta}(\mathbf{9 5} \% \mathbf{C I})$ \\
\hline $\begin{array}{l}\text { Total LPA time } \\
\text { Bouts }\end{array}$ & $0.288(-1.039$ to 1.615$)$ & $-0.197(-1.185$ to 0.791$)$ & $0.024(-0.043$ to 0.091$)$ \\
$15-30 \mathrm{~s}$ & $-0.006(-1.013$ to 1.002$)$ & $-0.925(-1.676$ to -0.175$) *$ & $0.003(-0.048$ to 0.054$)$ \\
$30-60 \mathrm{~s}$ & $2.180(0.357$ to 4.003$) *$ & $-1.246(-2.603$ to 0.112$)$ & $0.006(-0.098$ to 0.086$)$ \\
$1-3$ min & $2.822(0.681 \text { to } 4.964)^{*}$ & $-1.985(-3.579$ to -0.390$) *$ & $-0.001(-0.109$ to 0.107$)$ \\
$\geq 3$ min & $0.393(-0.042$ to 0.828$)$ & $-0.114(-0.438$ to 0.210$)$ & $0.013(-0.009$ to 0.035$)$ \\
\hline
\end{tabular}

Abbreviations: LPA, light physical activity; CI, confidence interval. adjusted analyses by age and socioeconomic status; ${ }^{\mathrm{b}}$ adjusted analyses by gender and socioeconomic status; ${ }^{\mathrm{c}}$ adjusted analyses by gender and age. ${ }^{*} P<0.05$.
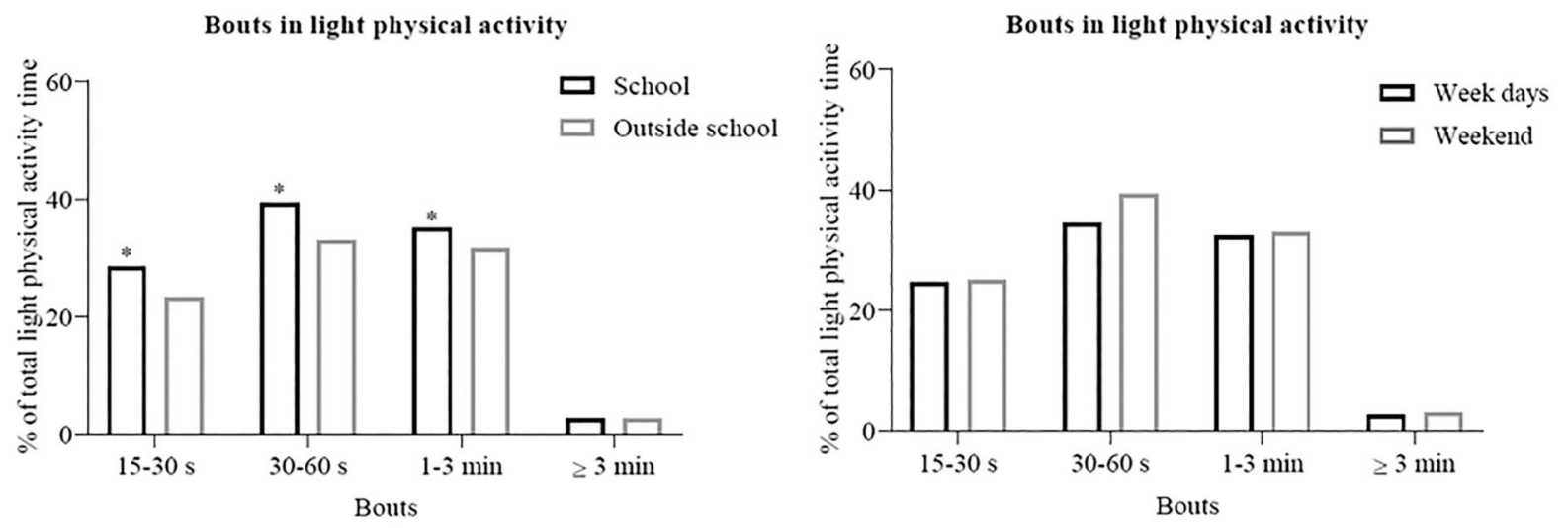

Figure 2 - Pattern of light physical activity according to bouts for weekdays or weekends and in or outside school. $* P<0.05$.

Our results showed that the daily time spent in LPA is considerably high (190 min or $22 \%$ of accelerometer wear time). In percentage values (of total time using accelerometer), our rates were similar to results found in literature, in which $27.5 \%{ }^{9}, 28.7 \%{ }^{13}$ were reported. However, in absolute terms, our estimates were lower than those observed in previous studies, which varied between 252 and $336 \mathrm{~min} /$ day $^{14,25}$. The variability observed among these estimates can be attributed to the different accelerometer thresholds adopted to quantify LPA, as well as to differences in the accelerometer wear time among participants of these studies. Nevertheless, it is noteworthy that adolescents tend to spend considerable daily time in LPA.

LPA time was predominantly accumulated from bouts between 15 seconds and three minutes, which is partially consistent with previous studies ${ }^{17,26}$, which pointed out that the LPA pattern was mostly accumulated in bouts of less than five minutes. This indicates that the LPA pattern seems to be more spontaneous than programmed, that is, it seems to reflect more transitions between sedentary behavior and LPA than the engagement in regular LPA practice. For example, in a previous study with the same sample, it was found that most of the sedentary time was accumulated in short bouts with a high number of breaks $^{5}$. Thus, considering that the daily fraction in LPA is higher than that accumulated in MVPA, it is believed that most sedentary behavior breaks are succeeded by short intermediate LPA bouts (i.e., sporadic household chores, personal hygiene, and others), thus forming this intermittent pattern ${ }^{27}$.

The LPA pattern was associated with gender, with female adolescents presented longer time in short and intermediate bouts compared to males. Inconsistent findings were observed in previous studies ${ }^{25,28}$, which do not found differences in total LPA volume between gender. However, our findings can be explained by the characteristics of activities performed by boys and girls. In this sense, girls appear to be more involved in household activities $^{29}$ which are predominantly characterized by light intensity (such as washing dishes, cooking, ironing) ${ }^{30}$. On the other hand, boys tend to spend longer daily time in MVPA $^{31}$. Thus, given the similarity in sedentary behavior between gender, the higher LPA in girls, in theory, does not appear to be a positive aspect for females.

The present study found that the LPA pattern was not associated with the economic level and did not differ according to the days of the week (weekdays and weekends), which contrasts with previous studies on physical activities performed at greater intensities. In a systematic review, Stalsberg et al. ${ }^{20}$ found that the time in MVPA is longer in children and adolescents belonging to the higher economic level, which seems to be the result of the easier 
access and engagement of these individuals to physical activities and/or supervised sports practices. Thus, the practice of structured sports traditionally occurs during weekdays, contributing to increasing MVPA estimates over that period ${ }^{21}$. On the other hand, light intensity activities, such as standing ${ }^{32}$, walking slowly, and performing household chores ${ }^{30}$ occur spontaneously and unstructured, being, therefore, less dependent on the economic condition and the context in which they are carried out.

The higher total time in LPA and the accumulation in very short, short and intermediate bouts during the school period was an interesting finding of the present study. Although the school environment favors the occurrence of sedentary behavior through high sitting time in the classroom, the pattern of this behavior is characterized by the predominance of short bouts $(<10 \mathrm{~min})^{33}$. Thus, this environment can also be very conducive to rapid interruptions of sedentary behavior ${ }^{5}$, which can occur both in the classroom (standing up to perform exercises in the classroom; to clarify doubts with teachers) and outside it (breaks between classes, recess, physical education classes). These breaks can be related to the transition from sedentary behavior to LPA, which can contribute to higher LPA accumulation in the school period.

Therefore, it is clear that the school environment, especially with regard to time in the classroom, contributes to a significant portion of sedentary time accumulated by children and adolescents. In this way, interventionist strategies implemented in the school period, such as the use of stand desks ${ }^{34}$, implementation of active breaks ${ }^{35}$, or the adoption of physically active lessons ${ }^{36}$ seem to be effective in reducing sitting time and promoting physical activity. Furthermore, it is important to promote LPA for other physical activity branches, especially for home and leisure PA, where opportunities for a sedentary lifestyle are increasingly frequent in the current daily life of adolescents.

Some limitations of the present study must be considered. Initially, a generalization of the results should be performed with caution, since the sample was exclusively composed of students from public schools. Sample loss (only $57 \%$ of individuals with valid accelerometer data) is also a limiting point. However, it should be highlighted that the sociodemographic characteristics (gender, age, socioeconomic status) were similar between individuals included and excluded from the analysis, which may have minimized any estimate bias. The LPA pattern in the school period was determined by analyzing data recorded by accelerometers during the day in which participants attended schools, although it was not possible to guarantee the attendance of students in the accelerometer monitoring time. In contrast, some positive points should be highlighted. The use of objective measures enabled estimating the LPA pattern with greater accuracy when compared to other methods used to measure physical activity. The use of a cutoff point in which the magnitude vector is considered allowed, in theory, greater precision in capturing the activities performed. The high average accelerometer wear time ( $\sim 14$ hours) allowed a detailed analysis of the LPA pattern during the awake period. To the best of our knowledge, this is one of the first studies to characterize the LPA pattern in Brazilian adolescents.

\section{Conclusion}

In conclusion, Brazilian adolescents spend more than three hours daily in light physical activity, being mostly accumulated in shorter bouts, from 30 seconds to 3 minutes. In addition, gender, age, and the school period appear to influence the LPA pattern in adolescents. Future studies should be carried out to analyze the association between LPA pattern and health outcomes in adolescents, as well as longitudinal changes in the LPA pattern during adolescence.

\section{References}

1. Poitras VJ, Gray CE, Borghese MM, Carson V, Chaput JP, Janssen I, et al. Systematic review of the relationships between objectively measured physical activity and health indicators in school-aged children and youth. Med Sci Sports Exerc. 2016;48(6):235-6. doi:10.1249/01. mss.0000485708.08247.c9.

2. Hibbing PR, Bassett DR, Coe DP, Lamunion SR, Crouter SE. Youth metabolic equivalents differ depending on operational definitions. Med Sci Sports Exerc. 2020;52(8):184653. doi: 10.1249/MSS.0000000000002299.

3. 2018 Physical Activity Guidelines Advisory Committee. 2018 Physical Activity Guidelines Advisory Committee Scientific Report. Washington, DC: U.S Department of Health and Human Services. 2018.

4. Hallal PC, Andersen LB, Bull FC, Guthold R, Haskell W, Ekelund U, et al. Physical Activity 1 Global physical activity levels: surveillance progress, pitfalls, and prospects. Lancet. 2012;380(9838):247-57. doi:10.1016/S0140-6736 (12)60646-1.

5. Ramos DE, Bueno MRO, Vignadelli LZ, Werneck AO, Ronque VER, Coelho-E-Silva MJ, et al. Pattern of sedentary behavior in brazilian adolescents. Rev Bras Atividade Física Saúde. 2018;23:1-6. doi:10.12820/rbafs.23e0009.

6. Carson V, Hunter S, Kuzik N, Gray CE, Poitras VJ, Chaput $\mathrm{JP}$, et al. Systematic review of sedentary behaviour and health indicators in school-aged children and youth: an update. Appl Physiol Nutr Metab. 2016;41(6):S240-65. doi:10.1139/apnm-2015-0630.

7. Amagasa S, Machida M, Fukushima N, Kikuchi H, Takamiya T, Odagiri Y. Is objectively measured light-intensity physical activity associated with health outcomes after adjustment for moderate-to-vigorous physical activity in adults? A systematic review. Int J Behav Nutr Physic Act. 2018;15(1):65. doi:10.1186/s12966-018-0695-z.

8. Ku PW, Hamer M, Liao Y, Hsueh MC, Chen LJ. Devicemeasured light-intensity physical activity and mortality: A 
meta-analysis. Scand J Med Sci Sport. 2020;30(1):13-24. doi:10.1111/sms.13557.

9. Harding SK, Page AS, Falconer C, Cooper AR. Longitudinal changes in sedentary time and physical activity during adolescence. Int J Behav Nutr Phys Act. 2015;12(1):1-7. doi:10.1186/s12966-015-0204-6.

10. Young DR, Hivert MF, Alhassan S, Camhi SM, Ferguson JF, Katzmarzyk PT, et al. Sedentary behavior and cardiovascular morbidity and mortality: A science advisory from the American Heart Association. Circulation. 2016;134(13): e262-79. doi:10.1161/CIR.0000000000000440.

11. Norton K, Norton L, Sadgrove D. Position statement on physical activity and exercise intensity terminology. J Sci Med Sport. 2010;13(5):496-502. doi:10.1016/j. jsams.2009.09.008.

12. Kwon S, Janz KF, Burns TL, Levy SM. Association between Light-Intensity Physical Activity and Adiposity in Childhood. Pediatr Exerc Sci. 2011;23(2):218-29. doi:10.1123/pes.23.2.218.

13. Dowd KP, Harrington DM, Hannigan A, Donnelly AE. Light-intensity physical activity is associated with adiposity in adolescent females. Med Sci Sports Exerc. 2014;46 (12):2295-300. doi:10.1249/MSS.0000000000000357.

14. Carson V, Ridgers ND, Howard BJ, Winkler EAH, Healy GN, Owen N, et al. Light-Intensity Physical Activity and Cardiometabolic Biomarkers in US Adolescents. PLoS One. 2013;8(8): e71417. doi:10.1371/journal.pone.0071417.

15. Moura BP, Rufino RL, Faria RC, Amorim PRS. Effects of isotemporal substitution of sedentary behavior with lightintensity or moderate-to-vigorous physical activity on cardiometabolic markers in male adolescents. PLoS One. 2019;14(11):1-13. doi:10.1371/journal.pone.0225856.

16. Grgic J, Dumuid D, Bengoechea EG, Shrestha N, Bauman A, Olds T, et al. Health outcomes associated with reallocations of time between sleep, sedentary behaviour, and physical activity: A systematic scoping review of isotemporal substitution studies. Int J Behav Nutr Phys Act. 2018;15 (1):1-68. doi:10.1186/s12966-018-0691-3.

17. Blaes A, Baquet G, Fabre C, Van Praagh E, Berthoin S. Is there any relationship between physical activity level and patterns, and physical performance in children? Int J Behav Nutr Phys Act. 2011;8(1):122. doi:10.1186/1479-5868-8122.

18. Stone MR, Rowlands AV, Middlebrooke AR, Jawis MN, Eston RG. The pattern of physical activity in relation to health outcomes in boys. Int J Pediatr Obes. 2009;4(4):30615. doi:10.3109/17477160902846179.

19. Telford RM, Telford RD, Olive LS, Cochrane T, Davey R. Why are girls less physically active than boys? Findings from the LOOK longitudinal study. PLoS One. 2016;11 (3):1-11. doi:10.1371/journal.pone.0150041.

20. Stalsberg R, Pedersen A V. Effects of socioeconomic status on the physical activity in adolescents: a systematic review of the evidence. Scand J Med Sci Sports. 2010:368-3. doi:10.1111/j.1600-0838.2009.01047.x

21. Brooke HL, Corder K, Atkin AJ, van Sluijs EMF. A Systematic Literature Review with Meta-Analyses of Withinand Between-Day Differences in Objectively Measured
Physical Activity in School-Aged Children. Sport Med. 2014;44(10):1427-38. doi:10.1007/s40279-014-0215-5.

22. ABEP - Associação Brasileira de Empresas de Pesquisa. Critério Padrão de Classificação Econômica Brasil. São Paulo, Brazil. 2010.

23. Toftager M, Kristensen PL, Oliver M, Duncan S, Christiansen LB, Boyle E, et al. Accelerometer data reduction in adolescents: effects on sample retention and bias. Int $\mathrm{J}$ Behav Nutr Physic Act. 2013; 10(1):40. doi: 10.1186/14795868-10-140.

24. Romanzini M, Petroski EL, Ohara D, Dourado AC, Reichert FF. Calibration of ActiGraph GT3X, Actical and RT3 accelerometers in adolescents. Eur J Sport Sci. 2014;14(1):91-9. doi:10.1080/17461391.2012.732614.

25. Telford RM, Telford RD, Cunningham RB, Cochrane T, Davey R, Waddington G. Longitudinal patterns of physical activity in children aged 8 to 12 years: The LOOK study. Int J Behav Nutr Phys Act. 2013;10:1-12. doi:10.1186/14795868-10-81.

26. Howie EK, Olds T, McVeigh JA, Abbott RA, Straker L. It's A-bout Time: Detailed Patterns of Physical Activity in Obese Adolescents Participating in a Lifestyle Intervention. J Phys Act Heal. 2015;12(11):1453-60. doi:10.1123/ jpah.2014-0480.

27. Corder K, Ekelund U, Steele RM, Wareham NJ, Brage S. Assessment of physical activity in youth. J Appl Physiol. 2008;105(3):977-87. doi:10.1152/japplphysiol.00094.2008.

28. Barker AR, Gracia-Marco L, Ruiz JR, Castillo MJ, Aparicio-Ugarriza R, González-Gross M, et al. Physical activity, sedentary time, TV viewing, physical fitness and cardiovascular disease risk in adolescents: The HELENA study. Int J Cardiol. 2018;254:303-9. doi:10.1016/j.ijcard.2017.11.080.

29. Kuo J, Schmitz KH, Evenson KR, et al. Physical and Social Contexts of Physical Activities Among Adolescent Girls. J Phys Act Health. 2009;6(2):144-52. doi: 10.1123/ jpah.6.2.144.

30. Ainsworth BE, Haskell WL, Whitt MC, Irwin ML, Swartz AM, Strath SJ, et al. Compendium of Physical Activities: an update of activity codes and MET intensities. Med Sci Sports Exerc. 2000;32(9 (Suppl 1)):S498-504. doi: 10.1097/ 00005768-200009001-00009.

31. Trost SG, Pate RR, Sallis JF, Freedson PS, Taylor WC, Dowda M, et al. Age and gender differences in objectively measured physical activity in youth. Med Sci Sports Exerc. 2001;34(2):350-5. doi: 10.1097/00005768-20020200000025 .

32. Butte NF, Watson KB, Ridley K, Zakeri IF, McMurray RG, Pfeiffer KA, et al. A Youth Compendium of Physical Activities: Activity Codes and Metabolic Intensities. Med Sci Sports Exerc. 2017:246-56. doi:10.1249/MSS.0000000000001430.

33. Costa BGG, Silva KS, Malheiros LEA, Minatto, G, Lima LRA, Petroski EL. Are adolescents really being sedentary or inactive when at school? An analysis of sedentary behaviour and physical activity bouts. Eur J Pediatr. 2018;177 (11):1705-10. doi: 10.1007/s00431-018-3233-8.

34. Minges KE, Chao AM, Irwin ML, Owen N, Park C, Whittemore $\mathrm{R}$, et al. Classroom standing desks and sedentary 
behavior: A systematic review. Pediatrics. 2016;137(2): e20153087. doi:10.1542/peds.2015-3087.

35. Masini A, Marini S, Gori D, Leoni E, Rochira A, Dallolio L. Evaluation of school-based interventions of active breaks in primary schools: A systematic review and meta-analysis. J Sci Med Sport. 2020;23(4):377-84. doi:10.1016/j. jsams.2019.10.008.

36. Norris E, Van Steen T, Direito A, Stamatakis E. Physically active lessons in schools and their impact on physical activity, educational, health and cognition outcomes: A systematic review and meta-analysis. Br J Sports Med. 2020;54 (14):826-38. doi:10.1136/bjsports-2018-100502.

\section{Corresponding author}

Leonardo Alex Volpato, Laboratório de Atividade Física e Saúde, Centro de Educação Física e Esportes, Rodovia
Celso Garcia Cid, km 380, Campus, P.O. box 6001, Londrina, PR, Brazil.

E-mail: leonardovolpato@uel.br.

Manuscript received on September 18, 2020

Manuscript accepted on February 22, 2021

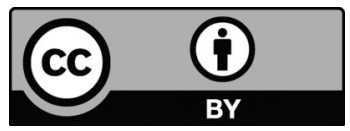

Motriz. The Journal of Physical Education. UNESP. Rio Claro, SP, Brazil - eISSN: 1980-6574 - under a license Creative Commons - Version 4.0 